\title{
Automated Detection of Diabetic Retinopathy for Early Diagnosis using Exudate Images
}

\author{
P. Manimegalai, S. Soundarya, J. R. Aswath, M. Sowmiya, N. Raja Lakshmi
}

\begin{abstract}
Retina plays a vital character in detection of various diseases in early point such as diabetes retinopathy which can be performed by analyzing the retinal images [6]. Diseased patients have to undergo periodic screening of eye. Standouts amongst the most predominant clinical indications of diabetic retinopathy are exudates [17]. To detect diabetic retinopathy in patients the ophthalmologist inspects the exudates by Ophthalmoscopy [17] where recognition of exudates is a vital diagnostic undertaking in which computer help may assume a noteworthy job. But intrinsic characteristics of retinal images detection process is difficult for the ophthalmologists. Here, we proposed another algorithm "Superpixel Multi-Feature Classification" for the programmed automatic recognition of retinal exudates successfully and to encourage ophthalmologist to give better patient finding experiencing diabetic retinopathy, advising them the level of seriousness ahead of time. The performance of algorithm has been compared as a result, the outcomes are effective and the sensitivity and specificity for our exudates identification is $80 \%$ and $91.28 \%$, respectively [15].
\end{abstract}

Keywords: Super pixel, Diabetic Retinopathy, Exudates, Image Processing.

\section{INTRODUCTION}

Diabetic retinopathy is a basic eye disease which can be viewed as sign of diabetes on the retina. Diabetic retinopathy is described by the improvement of retinal micro aneurysms, hemorrhages and exudates. Exudates happen when lipid or fat leaks from abnormal vein or aneurysms. Exudates are described by bright spots with sharp edges [17]. There are two sorts of diabetic retinopathy they are NPDR (no proliferative diabetic retinopathy) and PDR (proliferative diabetic retinopathy) where NPDR can be subdivided into mild NPDR,moderate NPDR, severe NPDR.Proliferative anyway alludes whether there is any neovascularization (strange vein development) present or not. This paper proposes another computer put together conclusion based with respect to the computerized picture preparing of exudate pictures so as to help individuals recognizing diabetic retinopathy ahead of time. The primary objective is to naturally group the non-proliferative diabetic retinopathy evaluation of any retinal picture. Our proposal has been tried on a database of 400 retinal pictures named by a 4-grade size of non-proliferative diabetic retinopathy.

Revised Manuscript Received on 14 August, 2019.

P. Manimegalai, Assoc.Professor, Instrumentation EnggDepartment,Karunya Institute of Tech and Sciences, Coimbatore, Tamilnadu, India.(Email: manimegalai@karunya.edu)

S. Soundarya, UG scholar/ECE,Department of ECEKAHE, Coimbatore, Tamilnadu, India.(Email: soundaryasakthi2@gmail.com)

J.R. Aswath, UG scholar/ECE,Department of ECEKAHE, Coimbatore, Tamilnadu, India.(Email: jraswath98@gmail.com)

M.Sowmiya, UG scholar/ECE,Department of ECEKAHE, Coimbatore, Tamilnadu, India.(Email: sowmiyamylsamy@gmail.com)

N. Raja Lakshmi, Associate Professor,Department of BiomedicalEngineering, KAHE, Coimbatore,Tamilnadu, India.(Email: praniraji1@gmail.com)
Accordingly, we acquired a most extreme sensitivity of $80 \%$ and a prescient limit estimation of $91.28 \%$. Robustness regarding changes in the parameters of the calculation has additionally been assessed. Amid the early long periods of research, image processing techniques, for example, thresholding, separating and morphological administrators were utilized. Recent research is focused on implementing segmentation, edge detection, mathematical modeling, classification, pattern recognition and texture analysis techniques.

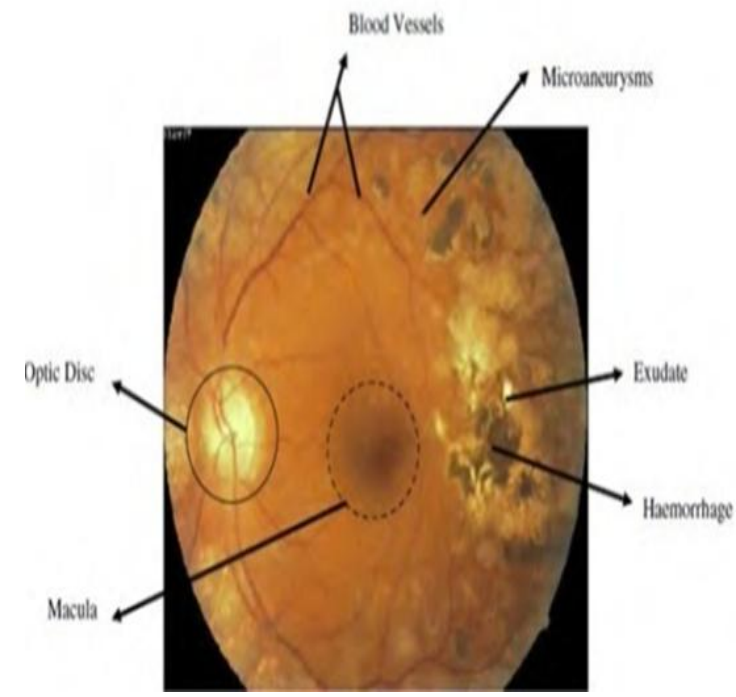

Fig1: Features in DR image

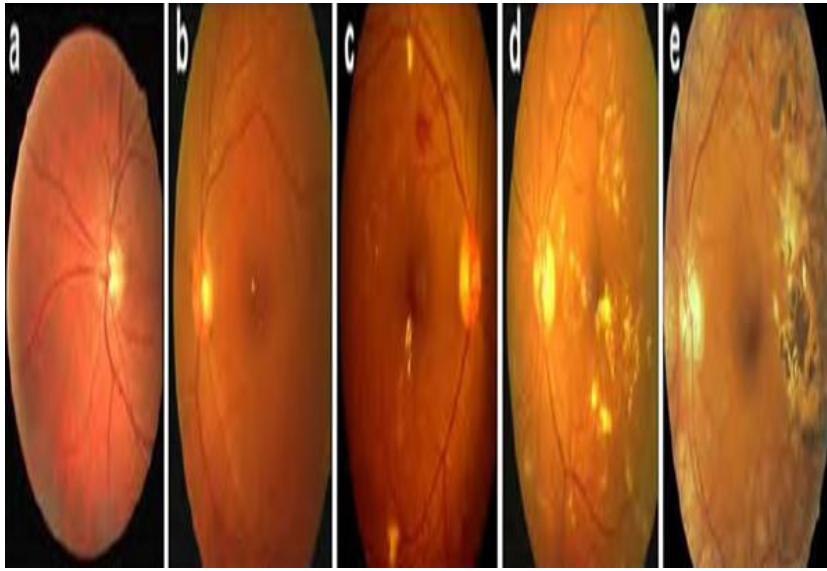

Fig 2: (a) Normal (b) Mild DR

(c) Moderate DR (d) Severe DR (e) PDR 


\section{II.RELATED WORKS}

Huiqi Li et.al., [2] has proposed an altered dynamic shape show in the shape discovery of optic plate; The achievement rates of circle restriction, circle limit recognition, and fovea limitation are 99\%, 94\%, and 100\%, separately. The affectability and particularity of exudates discovery are $100 \%$ and $71 \%$, correspondingly. The discovery and examination could be connected to programmed mass screening and finding of the retinal infections. Michael $\mathrm{d}$. Abramoff et.al.., [1]built up a strategy to assess the execution of a framework for computerized recognition of diabetic retinopathy in advanced retinal photos, worked from distributed calculations, in a huge, delegate, screening populace [4]. All out territory under the beneficiary working trademark bend was 0.84 , and nnm was 80 at an affectability of 0.84 and a particularity of 0.64 . Now, 7,689 of 10,000 tests had adequate picture quality, 4,648 of 7,689 (60\%) were genuine negatives, 59 of $7,689(0.8 \%)$ were false negatives, 319 of $7,689(4 \%)$ were genuine positives, and 2,581 of 7,689 (33\%) were false positives Desire Sidibé et.al.., [2] proposed a strategy for separating between various kinds of injuries is of enthusiasm for improving screening exhibitions. Utilization of inadequate coding systems for retinal pictures grouping is proposed. It accomplishes an affectability and an explicitness of $96.50 \%$ and $97.70 \%$ for the typical class; $99.10 \%$ and $100 \%$ for the drusen class; and $97.40 \%$ and $98.20 \%$ for the exudates class with a medium size word reference of 100 particles.

I.N. Figueiredoet.al., [3] proposed a novel computerized framework for the recognition and conclusion of these retinal sores by handling retinal supports pictures. Suitable paired classifiers for these three distinct kinds of sores are structured. This is performed by examining a few wavelet groups (coming about because of the isotropic un devastated wavelet change disintegration of the retinal picture green channel) and by utilizing a proper blend of Hessian multiscale investigation, variety division and animation surface deterioration.

\section{METHODOLOGY OF DETECTION}

\section{1. preprocessing}

Input image in RGB to Gray scale conversion which reduces the complexity in image analysis. In grayscale images, the watershed algorithm is easily conceptualized with the two spatial dimensions and one brightness dimension as a 3D image. There are two main steps used in the preprocessing stage, they are image resizing and ROI extraction. In this pre-processing is achieved through median filter and the region of interest extraction is carried out by adaptive $\mathrm{k}$ mean clustering

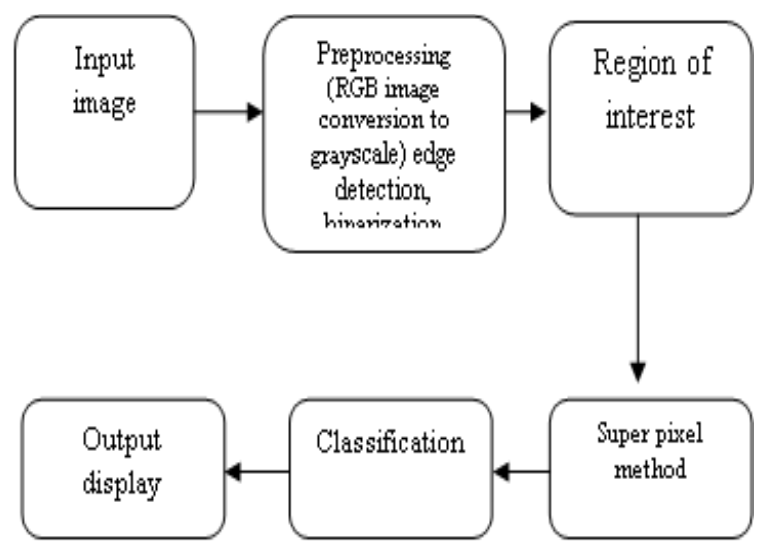

Fig3: Proposed block diagram for detection of Diabetic retinopathy
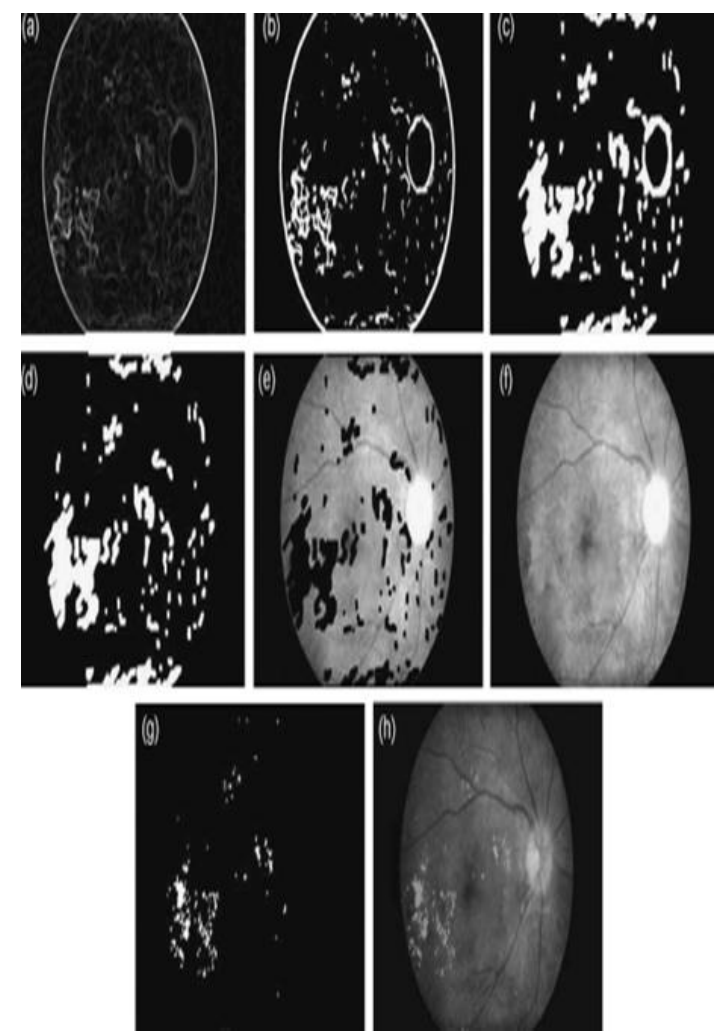

Fig.4: (a) Local variation image, (b) threshold image, (c) enclosed areas were flood-filled, (d) optic disc was

removed from image, (e) marker image, (f)

reconstructed image, $(\mathrm{g})$ difference image $(h)$ result superimposed on the original image.

\subsection{EDGE Detection}

In image processing the edge identification treats the localization of essential varieties of a gray level image and the identifies the physical and geometrical properties of objects [8]. At first it distinguishes diagrams of an item and limits In this undertaking objects and background in the image are separated. These features are used through advanced computer vision algorithms. These highlights are utilized through cutting edge computer vision algorithms. There are three distinct sorts of discontinuities in the

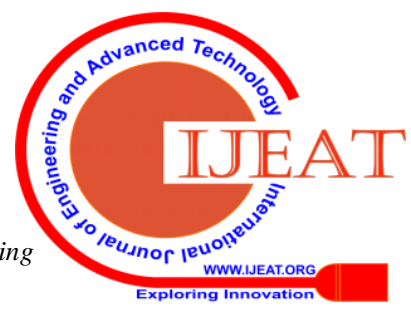


gray level, line and edges. Spatial covers identifies all the three sorts of discontinuities in the image.

Low Pass with Shaping Coefficient

Smoothing, sharpening, removing noise, and edge detection are done by image filtering [19]. Piece exhibit is connected to every pixel and its neighbors inside an image, the focal point of the part is lined up with the current pixel, and a square with an odd number $(3,5,7$, and so on.) of components in every dimension. Convolution process is utilized to apply channel to the picture in the spatial and recurrence space. At the point when the bit is focused over a pixel the initial segment of the convolution procedure duplicates the components of the portion by the coordinating pixel esteems. The components of the subsequent clustersare arrived at the midpoint of and the first pixel esteem is supplanted with the outcome. The CONVOL function is performed in this convolution procedure for an entire image.

\subsection{Binarisation}

The Sauvola technique for local binarization is utilized and we actualize it with tiling for efficiency. the threshold value is chosen close to the mean value, with very little contrast, and the threshold is chosen below the mean, by an amount proportional to the normalized local standard deviation.

\subsection{Region Of Interest}

In this the upper circle and lower circle in the eye is detected by the centroid values and matches the predefined values until the boundaries becomes equal the value is extended. Then the image is broken into pixels which are given as small images and multiple images to form a group. Based on these values masking of the image is done and the values such as area, perimeter, centroid and diameter are given as the result from the analyzed image.

\subsection{Superpixel Method}

\section{Superpixel Generation}

The effective graph based segmentation is used to generate the super pixels. In This technique limit between two locales are spoken to utilizing a chart based portrayal of the picture. Estimating the difference among the limit of the parts is in respect to a proportion of the divergence among neighboring components inside every one of the two components. This division strategy is quick to run and it creates not very coarse and not very fine division

\section{$>\quad$ Descriptors for Super Pixels}

The k-means algorithm defines the textons where textons are the cluster centers. In this 64 textons are used to describe the texture. In this study, the distance function for the super pixel color histogram distributions is chosen.

In this paper the $\chi 2$ is the separation used to figure the distinction between two shading histograms presented below. A couple of shading histograms $\mathrm{M}$ and $\mathrm{I}$, each contains $\mathrm{p}$ highlights and $\mathrm{n}$ receptacles absolutely and where, each element is partitioned into $\mathrm{n}$ canisters. At first the histogram is normalized to wipe out the impact from the distinctive size of the super pixels. The $\chi 2$ distance of the color histograms is defined to be,

$$
x^{2}(M, I)=\sum_{j=1}^{n p} \frac{\left(m_{j-} I_{J}\right) 2}{m_{j-}{ }^{I} J} \ldots \ldots \ldots \ldots
$$

(k-NN algorithm)

Let $\mathrm{x}$ be an unknown observation, which is a dimensional histogram data for a super pixel. suppose $\mathrm{L}$ is the number of classes, then

$$
k=\sum_{i=1}^{l} k_{i}
$$

The general rule for k-NN algorithm can be expressed as

$$
\begin{aligned}
& X \in l_{i,} \text { ifm } m_{i}(X) m_{j}(X), \text { forall } \neq i \\
m_{i}(X) & =k_{i} \ldots \ldots \ldots \ldots \ldots \ldots \ldots \ldots \\
m_{i}(X) & =\frac{\sum_{j=1}^{k i} 1 / D\left(X, X_{j}^{i}\right)}{\sum_{i=1}^{l} \sum_{j=1}^{k i}\left(X, X_{j}^{i}\right)} \ldots \ldots
\end{aligned}
$$

Where,

$D$ (x, xji) is the histogram distance between the unknown super pixel $\mathrm{x}$ and its $j$ th neighbour $\mathrm{j}$

belonging to class $l i$.

$\chi^{2}$ distance is used as the histogram distance function ( $\mathrm{j}) D$ (x, xji). [7]

\section{IV.RESULTS}

By using 400 model input images for testing we got these results on classification. In this way we have got descent results for our analysis. The performance of algorithm has been compared as a result, the results are effective and the sensitivity and specificity for our exudates identification is $80 \%$ and $91.28 \%$, respectively.

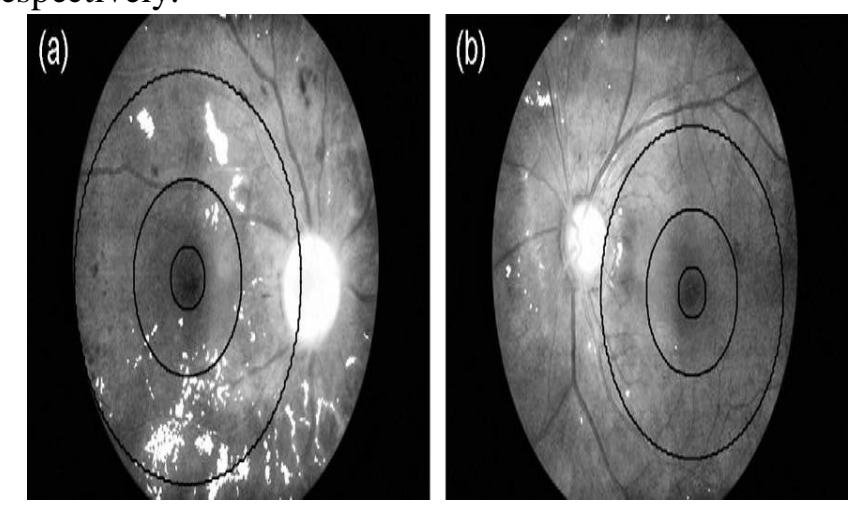

Fig5: (a) Macular grid fixated on the macular (b) superimposed on the exudate recognition result.[15]

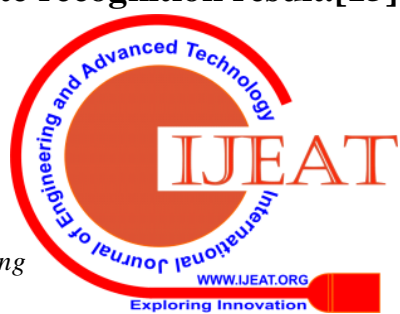




\section{AUTOMATED DETECTION OF DIABETIC RETINOPATHY FOR EARLY DIAGNOSIS USING EXUDATE IMAGES}

The automated diagnosis of diabetic retinopathy based on intensity of the retinal image is very successful in detecting exudates. It is not the final result application but it can be a preliminary diagnosis tool for ophtomologists.

\begin{tabular}{|l|l|l|}
\hline Method & $\begin{array}{l}\text { Sensitivity } \\
(\%)\end{array}$ & $\begin{array}{l}\text { Specificity } \\
(\%)\end{array}$ \\
\hline $\begin{array}{l}\text { Proposed } \\
\text { method puper pixel } \\
\text { (super } \\
\text { method) }\end{array}$ & 80 & 91.28 \\
\hline Gabor filter & 85.47 & 89.02 \\
\hline $\begin{array}{l}\text { Matched } \\
\text { filter }\end{array}$ & 83.79 & 88.21 \\
\hline
\end{tabular}

Table 1: comparison of the proposed method

\section{Output:}
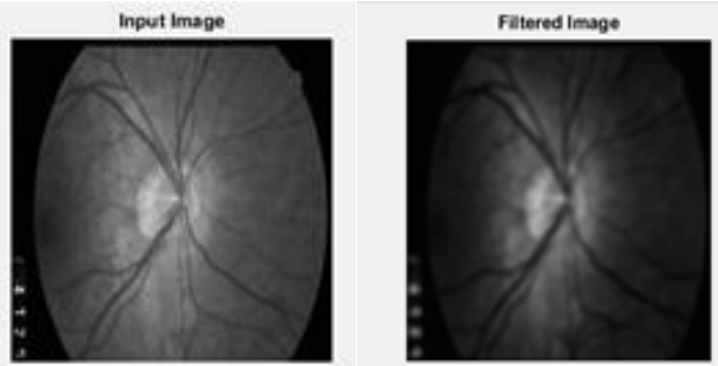

Fig :6 Input image Fig :7 Filtered image
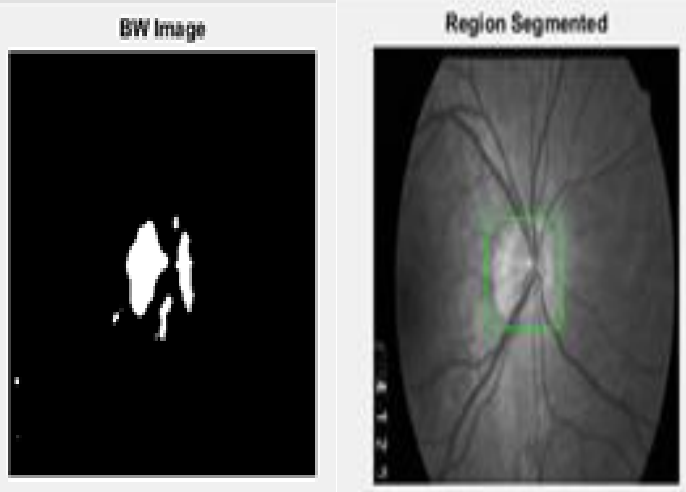

Fig 8: BW image Fig 9: Regionsegmented

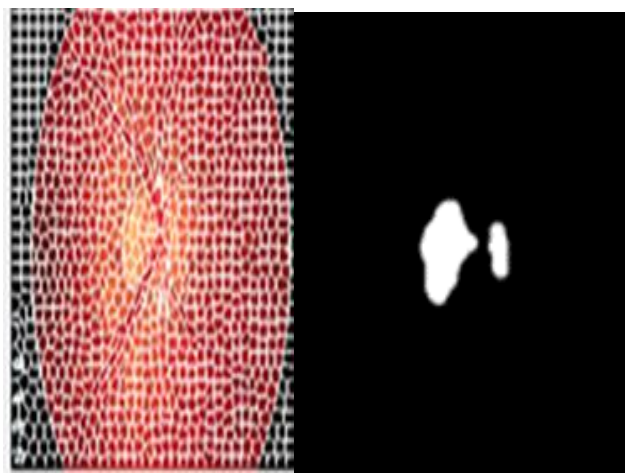

Fig10: SuperpixelmethodFig11: Background Detected

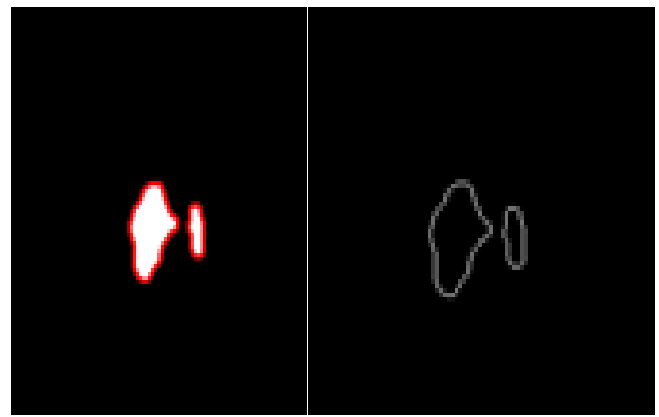

Fig 12: Blob DetectedFig 13: Boundary Detected

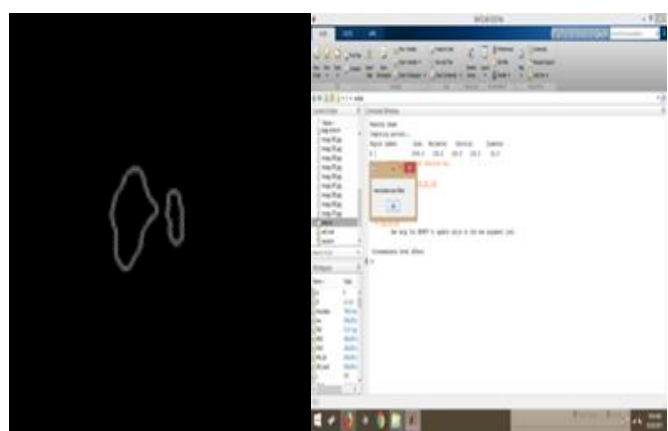

Fig 14: Skeleton ExtractFig 15: Final Result

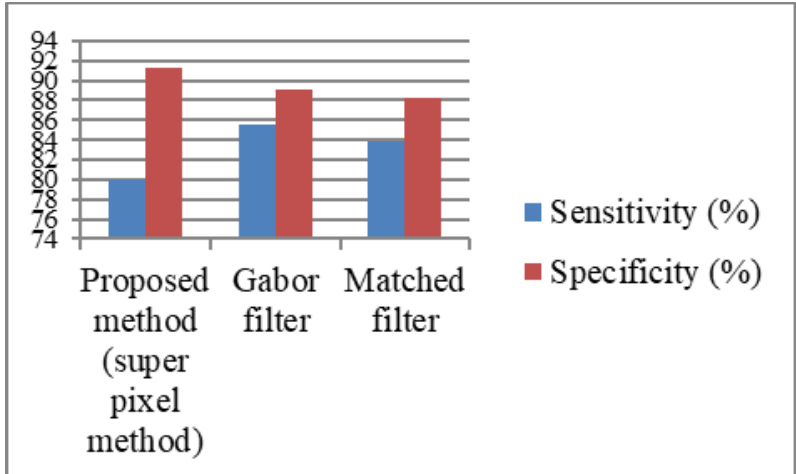

Table 2: Comparison of proposed method results on retinal database

\section{CONCLUSION}

In this paper, we set up a characterization framework dependent on the utilization of super pixels and the non-parametric $\mathrm{kNN}$ approach is embraced as thesuper pixel based order strategy, which is simple but robust. Thus by using the super pixel method the exudates size and intensity is found which advancely predicts the diabetic retinopathy [13]

\section{REFERENCES}

1. Sinthanayothin C, Boyce J.F, T. H. Williamson, H. L Cook, E. Mensah, S. Lal and D. Usher "Automated detection of diabetic retinopathy on digital fundus images" Diabetic. Medicine. 2002.

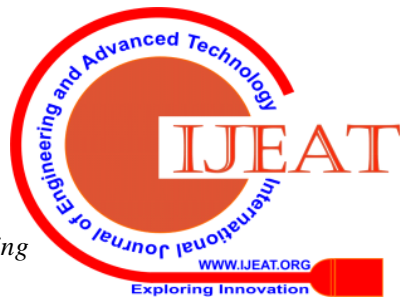


2. Huiqi $\mathrm{Li}^{*}$, OpasChutatape "Automated Feature Extraction in Colour Retinal Images by a Model Based Approach" IEEE TRANSACTIONS ON BIOMEDICAL ENGINEERING, 2, FEBRUARY 2004.

3. Jeyakkannan, N., and B. Nagaraj. "Online Monitoring of Geological Methane Storage and Leakage Based on Wireless Sensor Networks." Asian Journal of Chemistry 26 (2014).

4. Abràmoff MD1, Niemeijer M, Suttorp-Schulten MS, Viergever MA, Russell SR, van Ginneken B "Evaluation of a System for Automatic Detection of Diabetic Retinopathy from Colour Fundus Photographs in a Large Population of Patients with Diabetes" 2008 by the American Diabetes Association.

5. Akarasopharak, bunyarituyyanonvara, sarah barman, thomas h. Williamson. "Automatic detect ion of diabetic retinopathy exudates from non-dilated retinal images using mathematical morphology methods" computerized medical imaging and graphics, 2008.

6. Anirbankundu, rohitkamalchatterjee. "Retinal vessel segment at ion using morphological angular scale-space", 2012.Third international conference on emerging Applications of information technology,2012.

7. Guangyunzhang, xiupingjia, ngaim, Kwok. "Super pixel based remote sensing image. classification with histogram descriptors on spectral and spatial data", 2012 ieee international geoscience and remote sensing symposium, 2012.

8. Subodhkumar "fpga implementation of image segmentation by using edge Detection based on sobel edge operato" international journal of research in engineering and technology, 2013.

9. Carla Pereira, LuísGoncalves, Manuel Ferreira "Exudate segmentation in fundus images using an ant colony optimization approach" 2014 Elsevier Inc.

10. Rajalakshmi.N,Lakshmiprabha.V"Segregation of MRI images using hybrid evolutionary clustering algorithm", International journal of biomedical engineering and technology,2014.

11. DésiréSidibé, Ibrahim Sadek, FabriceMériaudeau "Discrimination of retinal images containing bright lesions using sparse coded features and SVM" 2015 Elsevier Ltd.

12. Natarajan,RajaLakshmiandViswanathan Lakshmi Prabha, "MRI brain image classification-a hybrid approach, International journal of imaging system and technology", Vol 25, PP-226-244, Sep2015.

13. Raju S. Maher, Sangramsing N. Kayte, MuktaDhopeshwarkar "Evaluation of a System for Automatic Detection of Diabetic Retinopathy from Colour Fundus Photographs for Screening Population" International Journal of Computer Applications (0975 8887) Volume 131 - No.3, December2015.

14. Enrique v. Carrera, Andres Gonzalez, Ricardo Carrera. "Automated detection of diabetic retinopathy using svm", 2017 ieee xxiv international conference on electronics, electrical engineering and computing(intercon), 2017.

15. Syed Ali GoharNaqvi n, Muhammad Faisal Zafar, IhsanulHaq "Referral system for hard exudates in eye fundus" 2015 Elsevier Ltd. C. Narmada, P. Manimegalai, S. Manimurugan, Journal of medical imaging and health informatics, a lossless compression scheme for grayscale medical images using a P2-Bit short technique, vol.7,19,2017.

16. Wei Zhou, chengdongwu, yugenyi, wenyoudu. "Automatic detection of exudates in digital colour fundus images using super pixel multi feature classification", ieee access, 2017.
17. Rd.badgujar, p.j. deore. "hybrid nature inspired smo-gbm classifier for exudate classification on fundus retinal images", irbm, 2019.

18. PoojaPandey,1.N.Sharma."Image Processing Techniques applied to satellite data for extracting lineaments using pciGeomatics and their morph tectonic interpretation in the parts of north western Himalayan frontal thrust", journal of the Indian society of remote sensing, 2019. 